\title{
Effects of Oxcarbazepine and Levetiracetam on Calcium, lonized Calcium, and 25-OH Vitamin-D3 Levels in Patients with Epilepsy
}

\author{
Duygu Aksoy, Betül Tekin Güveli, Pelin Doğan Ak, Hüseyin Sarı, Dilek Ataklı, Baki Arpacı \\ Department of Neurology, Bakirkoy Education and Research Hospital for Psychiatry, Neurology, and Neurosurgery, Istanbul, Turkey
}

\begin{abstract}
Objective: The primary objective of the present study was to further elucidate the effects of oxcarbazepine (OXC) and levetiracetam (LEV) monotherapies on the bone health status of patients with epilepsy.

Methods: This study included 48 patients who attended our epilepsy outpatient clinic, had a diagnosis of epilepsy, and were undergoing either OXC or LEV monotherapy and 42 healthy control subjects. The demographic and clinical features of the patients, including gender, age, onset of disease, daily drug dosage, and duration of disease, were noted. Additionally, the calcium, ionized calcium, and $25-\mathrm{OH}$ vitamin-D3 levels of the participants were prospectively evaluated.

Results: The 25-OH vitamin-D3, calcium, and ionized calcium levels of the patients taking OXC were significantly lower than those of the control group. These levels did not significantly differ between the patients taking LEV and the control group, but there was a significant negative relationship between daily drug dose and ionized calcium levels in the LEV patients.

Conclusion: In the present study, anti-epileptic drugs altered the calcium, ionized calcium, and 25-OH vitamin-D3 levels of epilepsy patients and resulted in bone loss, abnormal mineralization, and fractures. These findings suggest that the calcium, ionized calcium, and 25-OH vitamin-D3 levels of patients with epilepsy should be regularly assessed.
\end{abstract}

KEY WORDS: Antiepileptic drugs; Levetiracetam; Oxcarbazepine; 25-OH vitamin D3; Bone disease.

\section{INTRODUCTION}

Epilepsy is a common neurological disorder associated with recurrent seizures that, in some cases, requires life-long treatment. ${ }^{1,2)}$ Patients with epilepsy who are taking anti-epileptic drugs (AEDs) have a higher than normal risk of bone loss, abnormal mineralization, and fractures, but the exact pathogenic factors underlying these AEDassociated risks remain unclear. A variety of pathogenic mechanisms have been suggested, including the induction of hepatic enzyme activity with increases in the metabolism of 25-OH vitamin-D3, impaired calcium absorption, the direct effects of old generation AEDs on bone cells, resistance to parathyroid hormones, and an inhibition of calcitonin secretion. ${ }^{3-6)}$ Several large epidemiological studies found that patients with epilepsy have a two- to sixfold higher risk of fractures than the general population. ${ }^{7)}$ How-

\footnotetext{
Received: May 19, 2015/Revised: August 1, 2015

Accepted: August 25, 2015

Address for correspondence: Betül Tekin Güveli, MD

Department of Neurology, Bakirkoy Education and Research

Hospital for Psychiatry, Neurology, and Neurosurgery, Bakırköy

34147, Istanbul, Turkey

Tel: +90-532-7848273, Fax: +90-212-5729595

E-mail: betultekin2013@gmail.com
}

ever, the adverse effects of newer AEDs on bone metabolism are not yet sufficiently known. ${ }^{89}$ Thus, a determination of the presence of osteoporosis prior to the emergence of clinical signs is important so that measures can be taken to reduce the risk of fractures. It is crucial to evaluate the bone health of epileptic patients and to provide support with calcium and 25-OH vitamin-D3, if needed. ${ }^{6,10)}$

Thus, the present study analyzed the levels of serum calcium, ionized calcium, and 25-OH vitamin-D3, which are all important for bone metabolism, in patients using oxcarbazepine (OXC) or levetiracetam (LEV) monotherapies. The present results will aid in the assessment of bone health in patients with epilepsy who are prescribed these drugs as well as in the determination of the precautions necessary to maintain their health.

\section{METHODS}

\section{Participants}

The present study included 48 patients with epilepsy who had been using LEV or OXC monotherapy for at least 6 months and who volunteered for the study and 42 ageand sex-matched healthy volunteers as a control group.

(c) This is an Open-Access article distributed under the terms of the Creative Commons Attribution Non-Commercial License (http://creativecommons.org/licenses/by-nc/4.0) which permits unrestricted non-commercial use, distribution, and reproduction in any medium, provided the original work is properly cited. 
Table 1. Demographic and clinical characteristics of study participants

\begin{tabular}{cccc}
\hline Drug & Gender $(n)$, male/female & Age $(y r)$ & Duration of disease $(y r)$ \\
\hline Oxcarbazepine $(n=27)$ & $18 / 9$ & $37.63 \pm 10.20(27-48)$ & $10.64 \pm 8.96(0.5-41)$ \\
Levetiracetam $(n=21)$ & $8 / 13$ & $35.76 \pm 13.14(22-49)$ & $8.28 \pm 8.61(2-25)$ \\
Control group $(n=42)$ & $27 / 15$ & $32.71 \pm 7.20(25-49)$ & - \\
\hline
\end{tabular}

Values are presented as number only or mean \pm standard deviation (range).

The patients were attending the epilepsy outpatient clinic of Bakirkoy Education and Research Hospital for Psychiatry, Neurology and Neurosurgery (Istanbul, Turkey). Participants were excluded if they had a history of an overt medical disorder that could affect bone metabolism, had used medications known to affect bone turnover, had taken calcium or 25-OH vitamin-D3 supplements, had risk factors for osteoporosis (menopause or abnormal menstruation), or were pregnant.

Demographic data, including gender, age, type of epilepsy, type of seizure, duration of disease, duration of treatment, and drug dosage, were obtained. Venous blood samples were taken between AM 08:30 and 09:00 and then analyzed for calcium, ionized calcium, and $25-\mathrm{OH}$ vitamin-D3 levels. The optimal serum levels of calcium, ionized calcium, and 25-OH vitamin-D3 were considered to be 8.4-10.2 mg/dl, 4.2-5.6 mg/dl, and 20-80 ng/ml, respectively.

\section{Statistical Analysis}

All statistical analyses were performed using Number Cruncher Statistical System (NCSS) 2007 (NCSS LLC, Kaysville, UT, USA), Power Analysis and Sample Size (PASS; NCSS LLC), and Statistical Software 2008 (NCSS LLC). Student's $t$-tests were used to compare the quantitative data between two groups, a one-way analysis of variance (ANOVA) was used to compare the data of three or more groups, and Tukey's honest significant difference tests were used to determine differences among the groups. Mann-Whitney $U$-tests were used to compare two groups and Kruskal-Wallis tests were used to compare three or more groups in cases of groups that were not normally distributed. Mann-Whitney $U$-tests were also used to determine discrepancies among the groups. Pearson's chi-square tests were used to compare qualitative data, and Pearson's and Spearman's correlation analyses were used to analyze relationships among the parameters. $p$ values $<0.01$ and $<0.05$ were considered to indicate statistical significance.

\section{RESULTS}

The present study included 48 patients with epilepsy
Table 2. Calcium, ionized calcium, and $25-\mathrm{OH}$ vitamin-D3 levels in patients

\begin{tabular}{lcccc}
\hline & Gender & $\begin{array}{c}\text { OXC } \\
(n=27)\end{array}$ & $\begin{array}{c}\text { LEV } \\
(n=21)\end{array}$ & $\begin{array}{c}\text { Control group } \\
(n=42)\end{array}$ \\
\hline Calcium & $\mathrm{F}$ & $8.85 \pm 0.51$ & $8.83 \pm 0.49$ & $9.31 \pm 0.33$ \\
& $\mathrm{M}$ & $9.12 \pm 0.55$ & $9.18 \pm 0.34$ & $9.33 \pm 0.42$ \\
lonized calcium & $P^{*}$ & 0.225 & 0.124 & 0.587 \\
& $\mathrm{~F}$ & $4.21 \pm 0.18$ & $4.28 \pm 0.26$ & $4.43 \pm 0.18$ \\
& $\mathrm{M}$ & $4.35 \pm 0.17$ & $4.52 \pm 0.28$ & $4.53 \pm 0.25$ \\
& $P^{*}$ & 0.108 & 0.054 & 0.324 \\
& $\mathrm{~F}$ & $5.87 \pm 2.62$ & $5.95 \pm 2.16$ & $12.23 \pm 10.32$ \\
& $\mathrm{M}$ & $10.12 \pm 4.76$ & $10.38 \pm 5.97$ & $12.29 \pm 6.21$ \\
& $P^{*}$ & 0.081 & 0.074 & 0.431 \\
\hline
\end{tabular}

*Mann-Whitney U-test.

$M$, male; F, female.

(27 undergoing $\mathrm{OXC}$ monotherapy and 21 undergoing LEV monotherapy) and 42 healthy controls. The demographic and clinical features of the patients and the control participants are shown in Table 1. There were no statistically significant differences among patient and control groups in terms of mean age or sex $(p<0.05$; Table 1$)$.

The levels of calcium, ionized calcium, and $25-\mathrm{OH}$ vitamin-D3 did not differ significantly between males and females in either the patient or control group ( $p>0.05$; Table 2).

The levels of calcium and ionized calcium were normal in patients using $\mathrm{OXC}$, but they were lower than those of the control group $(p<0.01)$. The levels of calcium and ionized calcium did not differ significantly between patients using OXC or LEV and members of the control group ( $p>0.05$ ). However, the $25-\mathrm{OH}$ vitamin-D3 levels of both patient groups were lower than that of the control group (Table 3 ).

Among the patients using $\mathrm{OXC}$, there were no significant differences between the duration of drug usage, daily drug dosage, or ionized calcium or $25-\mathrm{OH}$ vitamin-D3 levels $(p>0.05)$. Among the patients using LEV, there were no significant differences between daily drug dosage or calcium or 25-OH vitamin-D3 levels ( $p>0.05$; Table 4).

However, there was a significant negative correlation between daily drug dosage and ionized calcium levels ( $\mathrm{r}$, -0.494 ; $p<0.05$; Table 5). 
Table 3. Calcium, ionized calcium, and $25-\mathrm{OH}$ vitamin-D3 levels

\begin{tabular}{|c|c|c|c|c|}
\hline & Oxcarbazepine $(n=27)$ & Levetiracetam $(n=21)$ & Control group $(n=42)$ & $p$ value \\
\hline Calcium $(8.4-10.2 \mathrm{mg} / \mathrm{dl})^{\star *}$ & $8.94 \pm 0.53$ & $9.01 \pm 0.46$ & $9.32 \pm 0.36$ & $0.003^{*}$ \\
\hline Ionized calcium $(4.2-5.6 \mathrm{mg} / \mathrm{dl})^{\star *}$ & $4.25 \pm 0.19$ & $4.41 \pm 0.29$ & $4.46 \pm 0.20$ & $0.006^{*}$ \\
\hline $25-\mathrm{OH}$ vitamin-D3 $(20-80 \mathrm{ng} / \mathrm{ml})^{* *}$ & $7.29 \pm 3.95$ & $8.69 \pm 5.28$ & $12.25 \pm 9.07$ & $0.011^{*}$ \\
\hline
\end{tabular}

${ }^{*} p<0.05,{ }^{* *}$ normal levels.

Table 4. Calcium, ionized calcium, and 25-OH vitamin-D3 levels and duration of drug usage

\begin{tabular}{lcrr}
\hline & & $\begin{array}{c}\text { OXC } \\
(n=27)\end{array}$ & $\begin{array}{r}\text { LEV } \\
(n=21)\end{array}$ \\
\hline Duration of drug usage and calcium & $r$ & -0.349 & -0.161 \\
& $p$ & 0.075 & 0.581 \\
Duration of drug usage and ionized & $r$ & -0.026 & -0.259 \\
calcium & $p$ & 0.905 & 0.315 \\
Duration of drug usage and 25-OH & $r$ & 0.356 & 0.043 \\
vitamin-D3 & $p$ & 0.068 & 0.853
\end{tabular}

OXC, oxcarbazepine; LEV, levetiracetam.

$r$, Spearman's correlation coefficient.

\section{DISCUSSION}

Because the risk of fractures in patients with epilepsy is two- to sixfold higher than that in the general population, it is thought that AEDs may negatively affect bone metabolism. ${ }^{10)}$ However, the adverse effects that AEDs exert on bone mineral metabolism have yet to be fully clarified. It has been suggested that these drugs affect the metabolism of 25-OH vitamin-D3 which, in turn, leads to reduced calcium absorption. The subsequent reduction in calcium levels could cause secondary parathyrotoxicosis that would negatively affect bone metabolism. On the other hand, the findings of several other studies suggest otherwise. ${ }^{11-13)}$

Only a few studies have investigated the relationship between new-generation AEDs and bone mineral metabolism. One of the important findings from the present study was that $25-\mathrm{OH}$ vitamin-D3 levels were low in both the patient and the control groups. It is known that weather can influence 25-OH vitamin-D3 levels such that they are highest at the end of summer and lowest at the end of winter. The fact that the blood samples used in the present study were obtained from participants at the end of winter may explain the low levels of 25-OH vitamin-D3. However, because the blood samples were taken from the patient and control groups during the same period, the findings of the study should not have been influenced by this variable. An individual's style of dress may also affect $25-\mathrm{OH}$ vitamin-D3 levels because clothes act as an important barrier between ultraviolet light and skin. For example, in Arabic countries, it is known that the traditional way of dressing
Table 5. Calcium, ionized calcium, and $25-\mathrm{OH}$ vitamin-D3 levels and age and total drug dose

\begin{tabular}{lcrc}
\hline & & $\begin{array}{c}\text { OXC } \\
(\mathrm{n}=27)\end{array}$ & $\begin{array}{c}\text { LEV } \\
(\mathrm{n}=21)\end{array}$ \\
\hline Total dose and calcium & $r$ & -0.223 & 0.114 \\
Total dose and ionized calcium & $p$ & 0.264 & 0.698 \\
Total dose and 25-OH vitamin-D3 & $r$ & -0.060 & -0.494 \\
& $p$ & 0.785 & $0.044^{*}$ \\
\hline
\end{tabular}

OXC, oxcarbazepine; LEV, levetiracetam.

$r$, Spearman's correlation coefficient; " $p<0.05$.

prevents the benefits of exposure to sunlight and can cause a 25-OH vitamin-D3 deficiency even though there is an abundant amount of sunlight. ${ }^{3)}$ In the present study, the participants' style of dress may have contributed to the low levels of 25-OH vitamin-D3 that were observed. Thus, the present finding that the control group exhibited a 25-OH vitamin-D3 deficiency demonstrates that appropriate exposure to sunlight is an important public health issue that must be taken seriously.

In the present study, the calcium, ionized calcium, and 25-OH vitamin-D3 levels of the patients using OXC were lower than those of the control group, which is compatible with previous findings. There were no correlations between daily $\mathrm{OXC}$ usage and calcium, ionized calcium, and 25-OH vitamin-D3 levels, but decreased calcium levels were associated with a longer duration of OXC usage; this trend was nearly statistically significant. It has been emphasized in the literature that an increased duration of drug usage lowers calcium levels. ${ }^{14-17)}$ Mintzer et al. ${ }^{18)}$ found that, depending on the dosage, patients using OXC exhibit significantly lower $25-\mathrm{OH}$ vitamin-D3 levels and suggested that there may be an inductive effect of OXC on cytochrome P450 or that it may affect osteoblast proliferation. Verotti et al. ${ }^{4)}$ reviewed the literature related to the effects of classic and novel AEDs on bone health and concluded that the abnormalities in calcium metabolism associated with these drugs result from their ability to induce the activity of cytochrome $\mathrm{P} 450$, which, in turn, causes reductions in 25-OH vitamin-D3 levels. However, the effects of many medications cannot be readily explained by 
25-OH vitamin-D3 metabolism. ${ }^{4)}$ And, in fact, a number of other reports have produced conflicting evidence. ${ }^{13,14,19)}$ Based on these and the present findings, it is suggested that follow-up visits and 25-OH vitamin-D3 replacement therapy should be mandatory for patients that have been on OXC treatment for a significant period of time because they may have low 25-OH vitamin-D3, calcium, and ionized calcium levels.

Only a few studies have investigated the effects of LEV on bone health, and the findings are controversial. Some of these studies found that LEV has no effect on bone health and that it does not affect serum calcium and $25-\mathrm{OH}$ vitamin-D3 levels. $^{20,21)}$

The calcium, ionized calcium, and $25-\mathrm{OH}$ vitamin-D3 levels of patients undergoing LEV monotherapy in the present study did not significantly differ from those of the control group. The fact that LEV is metabolized at a very low rate and that it does not have any influence on the cytochrome P450 enzyme system may be the reasons it does not negatively influence bone health. Additionally, there were no significant differences in the calcium, ionized calcium, and 25-OH vitamin-D3 levels of the present patients receiving LEV treatment according to duration of treatment, and only their ionized calcium levels decreased as the LEV dosage increased.

In a recent study, Nissen-Meyer et al. ${ }^{22)}$ found that LEV may change bone formation and quality without affecting bone mass and suggested that LEV may have a specific negative influence on trabecular bone structure rather than cortical bone structure. Although these authors could not exclude the possibility that the changes in bone structure were due to LEV-induced reductions in estrogen, they did not find any evidence of enhanced bone turnover related to estrogen deficiencies. In that rodent study, low-dose LEV caused a selective reduction in the biomechanical strength of the femoral neck, which represents metabolically active trabecular bone and the site where patients have an increased risk of fractures, without affecting bone mass and also resulted in reduced osteocalcin levels relative to controls. Because high-dose LEV did not impact bone parameters in that study, the authors suggested there may be a dose-dependent biphasic effect of LEV on bone. ${ }^{22,23)}$ Although relatively scarce, other studies investigating LEV have also shown that LEV does not affect trabecular bone. ${ }^{19)}$ This discrepancy may be attributed to differences in bone growth and metabolism between rats and humans and the fact that the dosages used in the rat studies were much higher $(50-150 \mathrm{mg} / \mathrm{kg}$ ) than the amounts used in human studies. ${ }^{23)}$ Thus, clinical epidemiological studies are necessary to clarify this issue in humans.

In a retrospective study, Beniczky et $a l .^{24)}$ observed a reduction in the bone mineral density of patients undergoing LEV treatment and suggested that these measurements should be considered for all adult patients receiving long-term LEV or OXC treatment. These findings suggest that increased dosages of LEV, and possibly other AEDS, may have a negative influence on the health of the bone structure.

Recent studies have indicated that annual measurements of 25-OH vitamin-D3 levels in patients using AEDs should be required and that this population may benefit from prophylactic $25-\mathrm{OH}$ vitamin-D3 treatment. ${ }^{25)}$ However, there is a lack of treatment guidelines regarding this issue.

In the present study, the mean calcium, ionized calcium, and 25-OH vitamin-D3 levels of patients undergoing OXC monotherapy were lower than those of the control group, whereas the values of the patients receiving LEV monotherapy were similar to those of the control group. These findings indicate that there is clinical value in regularly assessing the 25-OH vitamin-D3 levels of patients with epilepsy who are being treated with old- and new-generation AEDs and that this patient population may benefit from $25-\mathrm{OH}$ vitamin-D3 and calcium supplementation. Furthermore, it may be necessary to more closely assess the 25-OH vitamin-D3 and calcium levels of female patients in the peri- and postmenopausal periods as well as those of elderly patients and patients with any metabolic bone disorder. Additionally, these patients must be reminded to be conscious of maintaining a regular diet, engaging in physical exercise, exposing their bare skin to sunlight, and avoiding the risk factors of osteopenia, such as tobacco and alcohol.

\section{REFERENCES}

1. Hauser WA, Annegers JF, Rocca WA. Descriptive epidemiology of epilepsy: contributions of population-based studies from Rochester, Minnesota. Mayo Clin Proc 1996;71:576586.

2. Adams RD, Victor M, Ropper AH. Epilepsy and other seizure disorder. In: Adams RD, editor. Principles of neurology. 7th ed. New York:McGraw-Hill;2001. p.331-365.

3. Dawodu A, Kochiyil J, Altaye N. Pilot study of sunlight exposure and vitamin D status in Arab women of childbearing age. East Mediterr Health J 2011;17:570-574.

4. Verrotti A, Coppola G, Parisi P, Mohn A, Chiarelli F. Bone and calcium metabolism and antiepileptic drugs. Clin Neurol Neurosurg 2010;112:1-10.

5. Deckers CL, Genton P, Sills GJ, Schmidt D. Current limitations of antiepileptic drug therapy: a conference review. Epilepsy Res 2003;53:1-17.

6. Elliott JO, Jacobson MP. Bone loss in epilepsy: barriers to prevention, diagnosis, and treatment. Epilepsy Behav 2006; 8:169-175. 
7. Souverein PC, Webb DJ, Petri H, Weil J, Van Staa TP, Egberts T. Incidence of fractures among epilepsy patients: a population-based retrospective cohort study in the General Practice Research Database. Epilepsia 2005;46:304-310.

8. Pack AM, Morrell MJ. Adverse effects of antiepileptic drugs on bone structure: epidemiology, mechanisms and therapeutic implications. CNS Drugs 2001;15:633-642.

9. Pack AM, Morrell MJ. Epilepsy and bone health in adults. Epilepsy Behav 2004;5 Suppl 2:S24-S29.

10. Souverein PC, Webb DJ, Weil JG, Van Staa TP, Egberts AC. Use of antiepileptic drugs and risk of fractures: case-control study among patients with epilepsy. Neurology 2006;66:1318-1324.

11. Verrotti A, Greco R, Latini G, Morgese G, Chiarelli F. Increased bone turnover in prepubertal, pubertal, and postpubertal patients receiving carbamazepine. Epilepsia 2002;43:1488-1492.

12. Svalheim S, Røste LS, Nakken KO, Taubøll E. Bone health in adults with epilepsy. Acta Neurol Scand Suppl 2011; (191):89-95.

13. Miziak B, Błaszczyk B, Chrościńska-Krawczyk M, Danilkiewicz G, Jagiełło-Wójtowicz E, Czuczwar SJ. The problem of osteoporosis in epileptic patients taking antiepileptic drugs. Expert Opin Drug Saf 2014;13:935-946.

14. Babacan O, Karaoglu A, Vurucu S, Yesilkaya E, Yesilyurt $\mathrm{O}$, Cayci $\mathrm{T}$, et al. May long term oxcarbazepine treatment be lead to secondary hyperparathyroidism? J Clin Neurol 2012;8:65-68.

15. Cansu A, Yesilkaya E, Serdaroğlu A, Hirfanoğlu TL, Camurdan O, Gülbahar O, et al. Evaluation of bone turnover in epileptic children using oxcarbazepine. Pediatr Neurol 2008;39:266-271.

16. Bauer S, Hofbauer LC, Rauner M, Strzelczyk A, Kellinghaus $\mathrm{C}$, Hallmeyer-Elgner S, et al. Early detection of bone meta- bolism changes under different antiepileptic drugs (EDBoM-AED)--a prospective multicenter study. Epilepsy Res 2013;106:417-422.

17. Koo DL, Hwang KJ, Han SW, Kim JY, Joo EY, Shin WC, et al. Effect of oxcarbazepine on bone mineral density and biochemical markers of bone metabolism in patients with epilepsy. Epilepsy Res 2014;108:442-447.

18. Mintzer S, Boppana P, Toguri J, DeSantis A. Vitamin D levels and bone turnover in epilepsy patients taking carbamazepine or oxcarbazepine. Epilepsia 2006;47:510-515.

19. Cetinkaya Y, Kurtulmuş YS, Tutkavul K, Tireli H. The effect of oxcarbazepine on bone metabolism. Acta Neurol Scand 2009;120:170-175.

20. Koo DL, Joo EY, Kim D, Hong SB. Effects of levetiracetam as a monotherapy on bone mineral density and biochemical markers of bone metabolism in patients with epilepsy. Epilepsy Res 2013;104:134-139.

21. Lee R, Lyles K, Sloane R, Colón-Emeric C. The association of newer anticonvulsant medications and bone mineral density. Endocr Pract 2012;14:1-22.

22. Nissen-Meyer LS, Svalheim S, Taubøll E, Reppe S, Lekva $\mathrm{T}$, Solberg LB, et al. Levetiracetam, phenytoin, and valproate act differently on rat bone mass, structure, and metabolism. Epilepsia 2007;48:1850-1860.

23. Nissen-Meyer LS, Svalheim S, Taubøll E, Gjerstad L, Reinholt FP, Jemtland R. How can antiepileptic drugs affect bone mass, structure and metabolism? Lessons from animal studies. Seizure 2008;17:187-191.

24. Beniczky SA, Viken J, Jensen LT, Andersen NB. Bone mineral density in adult patients treated with various antiepileptic drugs. Seizure 2012;21:471-472.

25. Drezner MK. Treatment of anticonvulsant drug-induced bone disease. Epilepsy Behav 2004;5 Suppl 2:S41-S47. 